\title{
Update on the clinical utility of once-daily tacrolimus in the management of transplantation
}

\author{
This article was published in the following Dove Press journal: \\ Drug Design, Development and Therapy \\ I September 2014 \\ Number of times this article has been viewed
}

\author{
Maria Aurora Posadas Salas \\ Titte R Srinivas \\ Division of Nephrology and \\ Hypertension, Department of \\ Medicine, Medical University of South \\ Carolina, Charleston, SC, USA
}

\begin{abstract}
Adherence to immunosuppression and minimizing variability in drug exposure are important considerations in preventing rejection and maximizing overall transplant outcomes. The availability of once-daily tacrolimus may confer potential benefit by simplifying immunosuppressive regimens, thereby improving medication adherence among transplant recipients. Pharmacokinetic studies in healthy normal volunteers and stable transplant recipients suggest that once-daily tacrolimus is bioequivalent to twice-daily tacrolimus. Efficacy studies suggest that once-daily tacrolimus is noninferior to twice-daily tacrolimus with a concentration-dependent rejection risk. The incidence of biopsy-proven acute rejection, graft survival, and patient survival are more or less comparable between the two tacrolimus formulations. Once-daily tacrolimus has also been reported to have favorable effects on blood pressure, lipid profile, and glucose tolerance. Once-daily tacrolimus may be a viable option to consider for de novo immunosuppression or for conversion from conventional tacrolimus.
\end{abstract}

Keywords: pharmacokinetics, toxicity, immunosuppression, adherence

\section{Introduction}

Immunosuppression management in clinical transplantation aims to balance delivery of efficacy against adverse reactions using therapeutic drug monitoring. Another important aspect of managing immunosuppression is ensuring compliance by the transplant patient in order to sustain an allograft free from rejection. ${ }^{1}$ Overall acceptability of a regimen, convenience of dosing, and tolerability of individual drugs or regimens frequently impact patients' medication compliance. ${ }^{2}$ Hence, most studies on immunosuppressive agents focus on efficacy and safety, with the goal of achieving the best possible allograft function with minimal adverse effects. Over the years, immunosuppressive regimens used in transplantation have evolved. In current practice, the most commonly used immunosuppressive regimen includes a calcineurin inhibitor (cyclosporine, tacrolimus) and an antimetabolite (mycophenolate mofetil, azathioprine), with or without corticosteroids. ${ }^{3}$ Increasing availability of a broad range of immunosuppressive medications and formulations opens possibilities of designing regimens that achieve the best possible graft outcome along with minimal adverse effects while, at the same time, maximizing treatment adherence among transplant recipients.

\section{Rationale for once-daily tacrolimus in clinical practice}

Conventional immunosuppression in the modern era involves twice-daily oral dosing of calcineurin inhibitors and antiproliferatives such as mycophenolate mofetil. 
This need for twice-daily dosing schedules has been associated with nonadherence. Previous reviews directed at recipients transplanted between the late 1980s and mid-2000s showed that the prevalence of nonadherence to immunosuppressive medications averaged about $25 \%$. $^{2}$ This nonadherence to medications was felt to contribute to about $20 \%$ of late acute rejection episodes and $16 \%-36 \%$ of graft losses. ${ }^{2}$ It would therefore be reasonable to expect that a simplified immunosuppressive regimen that involves less frequent drug dosing would improve both patients' medication compliance and long-term allograft outcomes. ${ }^{1,4}$ The recent availability of once-daily tacrolimus formulation could thus offer the potential benefit of improved medication compliance and possibly better allograft outcomes by decreasing pill burden and thereby simplifying dosing schedule. Since there is more extensive experience in the use of the twice-daily tacrolimus formulation in clinics, the increased utilization of once-daily tacrolimus demands a review of evidence that this formulation is comparable, or noninferior if not superior, to twicedaily tacrolimus in terms of safety and efficacy.

\section{Once-daily tacrolimus: dosing and pharmacokinetics}

A once-daily tacrolimus formulation has recently been developed and licensed for use. ${ }^{5}$ Compared to twice-daily tacrolimus, once-daily tacrolimus is typically released more distally in the gastrointestinal tract by virtue of its dissolution properties. It has the same active component and metabolism as twice-daily tacrolimus. Once-daily tacrolimus was initially developed as modified release- 4 tacrolimus, using varying concentrations of additives such as ethylcellulose, hypromellose, and lactose monohydrate that alter water penetration into the formulation. Recommended doses for once-daily tacrolimus start at $0.2-0.3 \mathrm{mg} / \mathrm{kg} /$ day within 24 hours of kidney transplantation and $0.1-0.2 \mathrm{mg} / \mathrm{kg} /$ day within $12-18$ hours of liver transplantation. ${ }^{5}$

In a randomized, four-way, crossover trial conducted in six healthy white male subjects, various formulations of tacrolimus were administered to be activated in the stomach, proximal small intestines, distal small intestines, and ascending colon, and were tracked using radiolabeled material. Results showed that tacrolimus was absorbed in the small intestines as well as the colon. Pharmokinetic (PK) studies showed that, although there was a substantial intersubject variation in $\mathrm{PK}$ parameters, there were no statistical differences in the peak concentration $\left(\mathrm{C}_{\max }\right)$ and areas under the curve (AUCs) achieved with the different tacrolimus regimens released at various sites of the gastrointestinal tract. ${ }^{6}$
Phase I PK studies among healthy volunteers showed that once- and twice-daily tacrolimus administered based on a milligram-to-milligram conversion achieved similar AUC and trough levels (minimum concentration $\left.\left[\mathrm{C}_{\text {min }}\right]\right)^{5}$ Once-daily tacrolimus was noted to have a lower $\mathrm{C}_{\max }^{5,7}$ and a longer time to achieve maximal concentration $\left(\mathrm{T}_{\max }\right)$ compared to twice-daily tacrolimus..$^{5}$ The effects of food intake on the PK profile of once- and twice-daily tacrolimus are similar in that $\mathrm{C}_{\max }$ and AUC decreased by about $20 \%-25 \%$, while $\mathrm{T}_{\max }$ increased by $80 \%{ }^{5}$

Phase II PK studies involving adult kidney, liver, and heart transplant patients showed that the AUC and trough levels achieved with once-daily tacrolimus were within the $80 \%-125 \%$ range in comparison with twice-daily tacrolimus, suggesting bioequivalence in PKs between the two tacrolimus formulations. ${ }^{5}$ However, slightly lower AUC and trough levels were observed in patients who received once-daily tacrolimus, necessitating higher doses of the once-daily formulation to achieve a given trough level. ${ }^{5}$ In a Japanese study that compared once- with twice-daily tacrolimus in 102 de novo kidney transplant recipients, PK studies revealed similar AUC, $\mathrm{C}_{\max }$, and trough levels between the two formulations. The trough level correlated well with AUC ( $R^{2}=0.94$ for once-daily tacrolimus and $R^{2}=0.93$ for twice-daily tacrolimus). ${ }^{8}$

In a Phase II study of 47 stable kidney transplant patients converted from twice- to once-daily tacrolimus, PK studies done on days 7 (baseline prior to conversion), 14 , and 21 revealed significantly lower $C_{\max }$, peak-trough ratio, and percent fluctuation as well as significantly longer $\mathrm{T}_{\max }$ for once-daily tacrolimus compared to the twice-daily formulation. ${ }^{9}$ Similar observations were noted in 57 liver transplant recipients converted from twice- to once-daily tacrolimus. ${ }^{10}$ The greater bioavailability of once-daily tacrolimus allowed similar AUC exposure using a dose that is about $30 \%$ lower than the twice-daily formulation. ${ }^{9,10}$ Among pediatric kidney transplant recipients, AUC and trough were noted to decrease after conversion to once-daily tacrolimus. ${ }^{11,12}$ These differences were statistically significant in a PK study involving 34 patients $^{11}$ and not statistically significant in another study involving 19 patients. ${ }^{12} \mathrm{C}_{\max }$ was also not significantly different between formulations and CYP3A5 genotypes. ${ }^{12}$ PK studies conducted on 19 stable lung transplant patients converted to once-daily tacrolimus showed that the bioavailability of tacrolimus is similar with once- and twice-daily formulations. AUC and trough levels were similar in both formulations. ${ }^{13}$ Based on the above, it is recommended that tacrolimus be taken on an empty stomach at least 1 hour 
before or 2-3 hours after a meal. Given the overall similarities in the PK profile of both tacrolimus formulations, therapeutic drug monitoring for both once- and twice-daily tacrolimus is done based on the $\mathrm{C}_{\min }$ or trough level, measured 24 hours from the last once-daily tacrolimus dose and 12 hours after the twice-daily tacrolimus dose. ${ }^{5}$ Table 1 summarizes the PK characteristics of once-daily tacrolimus in comparison with the twice-daily formulation.

The pharmocokinetics of both tacrolimus formulations are affected by patient age, race, hematocrit, and cytochrome P450 (CYP) 3A5 genotype. Between-subject PK variability is similar between once- and twice-daily tacrolimus. However, within-subject inter-occasion PK variability, a measure of stability of drug exposure over time, is lower with once-daily tacrolimus. ${ }^{5}$ PK studies performed on 40 kidney transplant recipients converted from twice- to once-daily tacrolimus revealed that conversion to the once-daily formulation decreased intrapatient variability. ${ }^{14}$

Previous pharmacogenetic studies demonstrated that Caucasian and East Asian patients with CYP3A5*1 allele achieved twofold lower dose-normalized tacrolimus blood concentrations compared to CYP $3 \mathrm{~A} 5 * 3 / * 3$ homozygotes. ${ }^{15}$ $\mathrm{C}_{\max }$ was not significantly different between formulations and CYP3A5 genotypes. ${ }^{16}$ Stifft et al reported that patients with CYP $3 \mathrm{~A} 5 * 1 / * 3$ genotype had a greater decrease in intrapatient variability after conversion to once-daily tacrolimus compared to patients with $\mathrm{CYP} 3 \mathrm{~A} 5 * 3 / * 3$ genotype, although the differences did not reach statistical significance. ${ }^{14}$ In a PK study on 97 Japanese kidney transplant recipients, absolute bioavailability was noted to be lower in CYP3A5*1 genotype patients compared to those with the CYP3A $5 * 3 / * 3$ genotype, while relative bioavailability was lower in patients on once-daily tacrolimus compared to those on twice-daily tacrolimus, regardless of CYP3A5 genotype. ${ }^{16}$ In a similar study among Taiwanese kidney transplant recipients, ${ }^{17} \mathrm{PK}$ assessment was performed in 71 patients with high-expressive genotype (CYP $3 \mathrm{~A} 5 * 1 / * 1$ or CYP3A5*1/*3) and 79 patients with low-expressive genotype (CYP3A5*3/*3). Results showed that the tacrolimus trough levels were significantly higher in patients with low-expres-

Table I Pharmacokinetic profile of once-daily tacrolimus compared to twice-daily tacrolimus

Lower maximum concentration $\left(\mathrm{C}_{\max }\right)$

Longer time to peak concentration $\left(\mathrm{T}_{\max }\right)$

Area under the curve and trough within $80 \%-125 \%$ of twice-daily tacrolimus

Lower intrapatient variability sive genotype before and after conversion to the once-daily formulation. As could be expected, tacrolimus dose was significantly higher in the high-expressive genotype group both before and after conversion to once-daily tacrolimus. Conversion from twice- to once-daily tacrolimus resulted in a decrease in variability in the tacrolimus trough levels among patients with high-expressive genotype. ${ }^{17}$

Despite a lack of studies involving drug interactions with once-daily tacrolimus, it is reasonable to believe that onceand twice-daily tacrolimus would exhibit similar drug interaction patterns as they share the same active constituent. ${ }^{5}$

The similarity in the PK profiles of once- and twice-daily tacrolimus suggests that the two tacrolimus formulations are bioequivalent. Both formulations have similar AUC and trough levels, although there was a trend toward lower, although often not statistically significant, $\mathrm{AUC}$ and $\mathrm{C}_{\max }$ with once-daily tacrolimus. Once-daily tacrolimus could allow for minimization in intrapatient inter-occasion variability in $\mathrm{C}_{\min }$, especially among patients with CYP3A5 high-expressive genotype.

Whether the PK bioequivalence between the two tacrolimus formulations translates to the noninferiority of oncedaily tacrolimus compared to twice-daily tacrolimus can only be determined by efficacy studies that compare outcomes of patients who received either formulation; these are discussed in the following section.

\section{Efficacy studies on once-daily tacrolimus Kidney transplantation}

A randomized, open-label, multicenter, three-armed noninferiority trial ${ }^{18}$ involving 638 de novo kidney transplant recipients, comparing the efficacy of once- and twice-daily tacrolimus with cyclosporine, showed that both tacrolimus formulations were noninferior to cyclosporine, as measured by efficacy failure rate, consisting of death, graft failure, and biopsy-proven acute rejection (BPAR) at 1 year. The rates of BPAR at 6 months and 1 year were significantly lower in the twice-daily tacrolimus group (3.8\% and $7.5 \%$, respectively) compared to the cyclosporine group (11.8\% and $13.7 \%$, respectively). BPAR rates at 6 months and 1 year were not statistically different between the once-daily tacrolimus $(7.9 \%$ and $10.3 \%$, respectively) and cyclosporine groups. ${ }^{18} \mathrm{~A}$ greater proportion of patients receiving once-daily tacrolimus had multiple episodes of rejection that required anti-lymphocyte treatment. ${ }^{5,18}$ It is worth noting that about one-third of the patients who received once-daily tacrolimus had trough levels below target during the early post-transplant period 


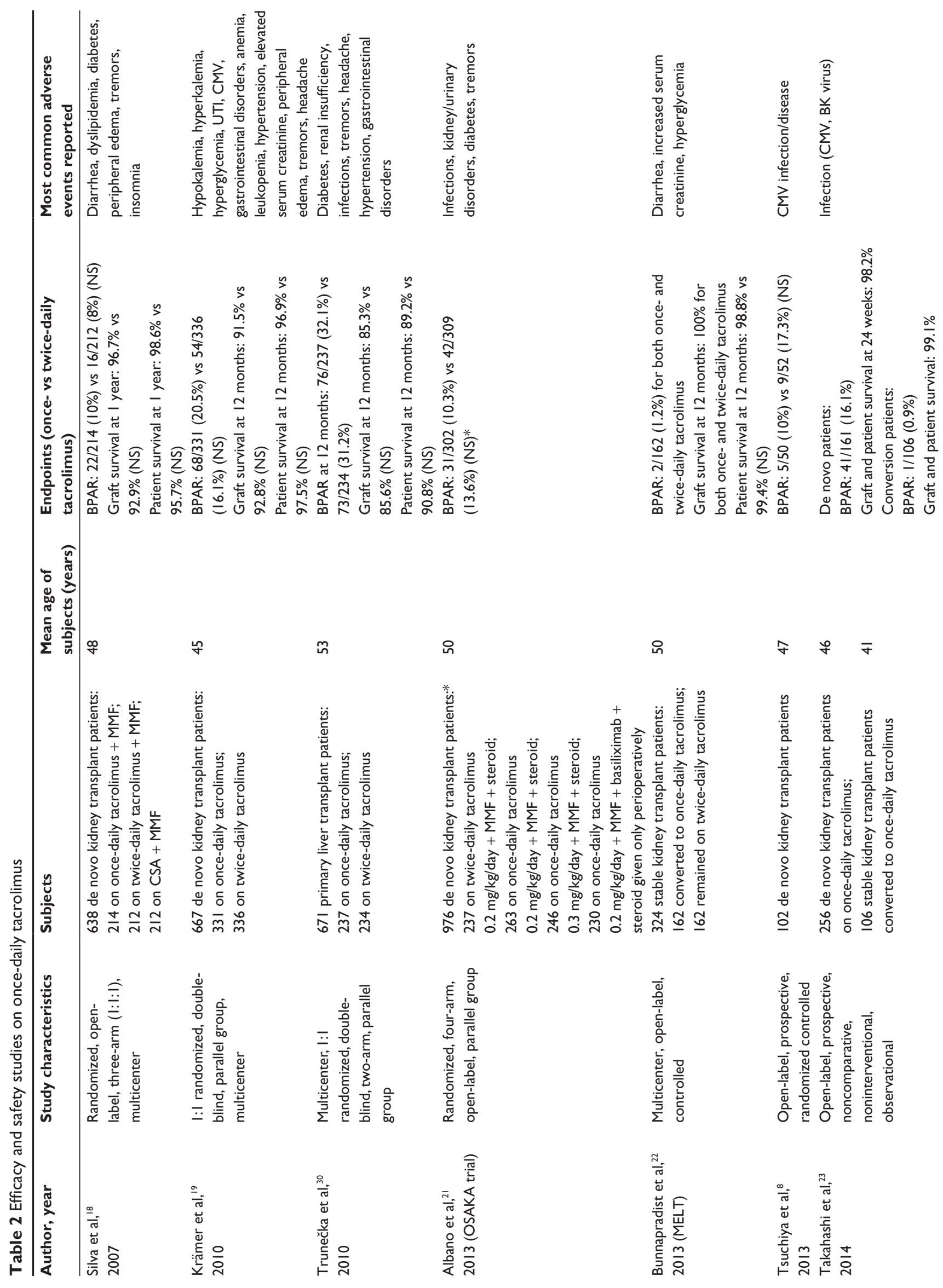



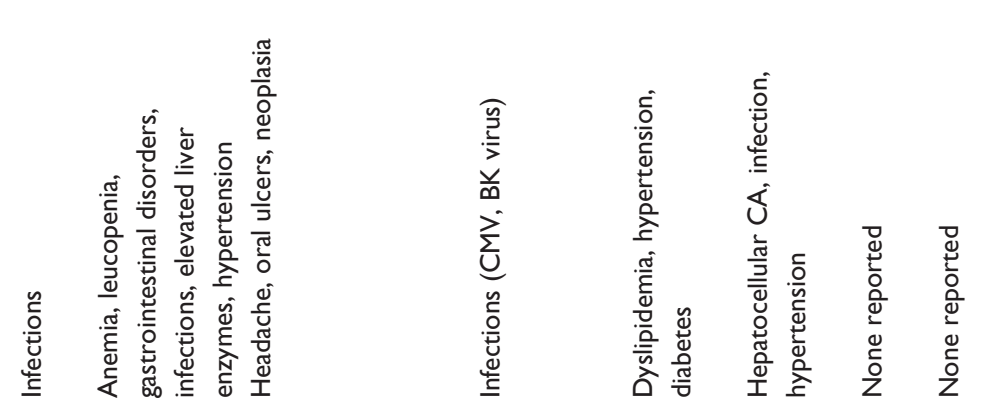

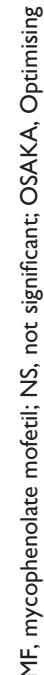

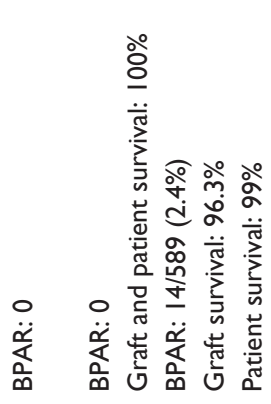

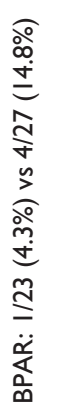

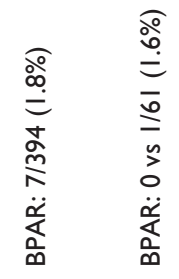

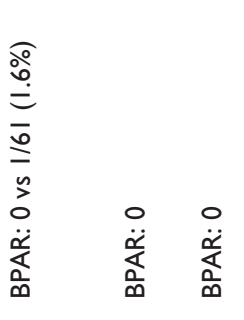

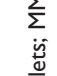

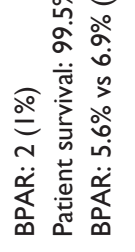

$\infty$

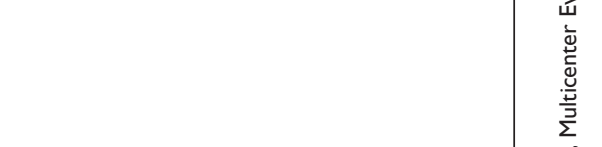

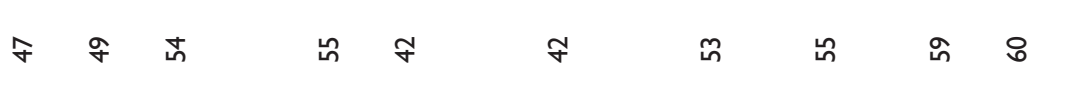

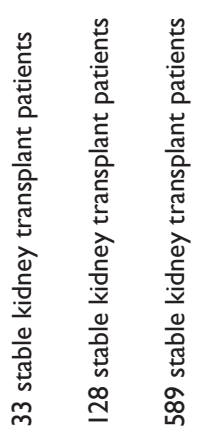
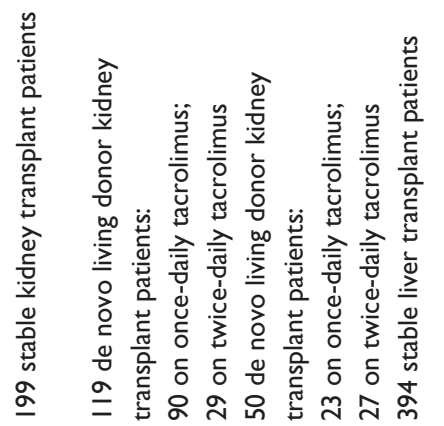

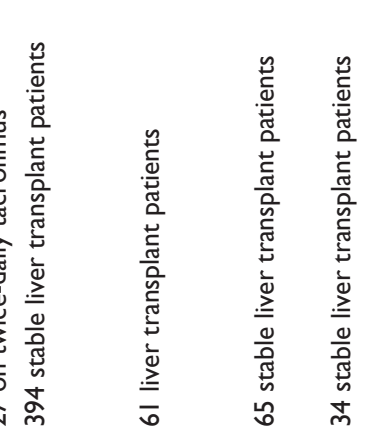
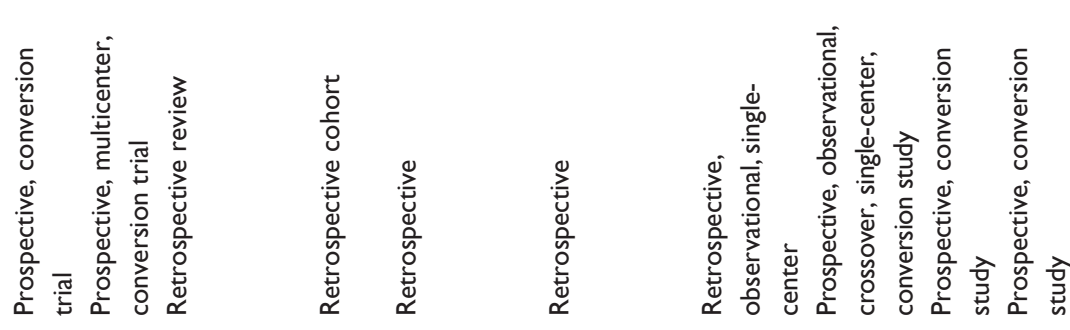

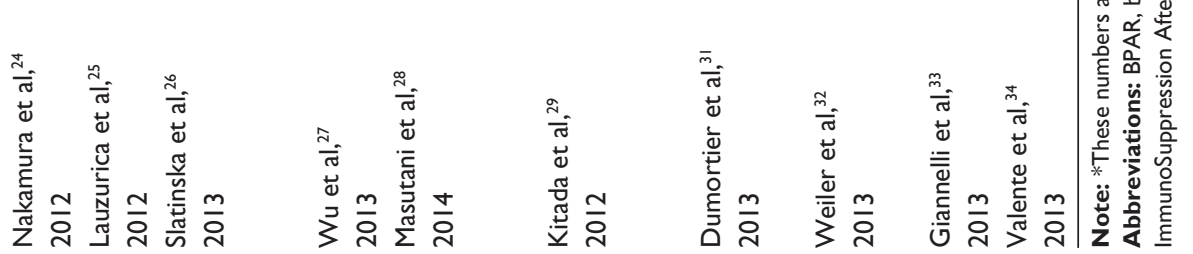


(day 3). Overall, across all three treatment groups, the mean tacrolimus trough levels achieved at days 3 and 30 were found to be statistically similar between patients who developed and did not develop BPAR. Nevertheless, there was a trend toward higher mean trough levels achieved among patients in the twice-daily tacrolimus group who did not develop BPAR compared to those who developed BPAR on either tacrolimus formulation.

Another randomized, double-blind, multicenter noninferiority trial with 667 de novo kidney transplant patients compared the efficacy of once-daily tacrolimus with twicedaily tacrolimus. All subjects received mycophenolate mofetil and corticosteroids, without antibody induction. Biopsies were evaluated by a local histopathologist (local biopsy) and reviewed by an independent, blinded pathologist for verification (central biopsy). The primary endpoint was local BPAR within 24 weeks post-transplant. Secondary endpoints included local BPAR rate at 12 months, central BPAR rates, graft loss, or death. In this trial, the once-daily tacrolimus group was found to have slightly higher BPAR rates at 24 weeks and 12 months. ${ }^{19}$ Although the differences in local and central BPAR rates at 24 weeks and 12 months between once- and twice-daily tacrolimus did not reach statistical significance, overall central BPAR rate for once-daily tacrolimus was significantly higher compared to twice-daily tacrolimus. It is notable that the treatment differences between once- and twice-daily tacrolimus fell outside the $10 \%$ prespecified noninferiority margin for the primary and secondary endpoints, suggesting that once-daily tacrolimus may not be noninferior to twice-daily tacrolimus. Moreover, there were five reported cases of Banff III rejection in the once-daily tacrolimus group, although these were deemed to be of a lower grade rejection and/or different pathology on central review. ${ }^{20}$ It is also worth noting that the mean tacrolimus trough achieved in the oncedaily tacrolimus group during the first week post-transplant was significantly lower compared to the twice-daily tacrolimus group. Whether this relatively lower tacrolimus trough early post-transplant in the once-daily tacrolimus group contributed downstream to higher BPAR rates deserves further investigation. Even more importantly, future experience will need to verify whether this rejection risk may be modifiable by optimizing drug exposure with increase in the daily dose of the drug. Overall, results showed comparable patient and allograft survival, renal function, and adverse effects between the two formulations. ${ }^{5,19}$

In an open-label, randomized study (Optimising ImmunoSuppression After Kidney Transplantation with ADVAGRAF [OSAKA] trial), 976 adult kidney transplant patients were grouped into four arms to receive: twice-daily tacrolimus
$0.2 \mathrm{mg} / \mathrm{kg} /$ day (arm 1); once-daily tacrolimus $0.2 \mathrm{mg} / \mathrm{kg} / \mathrm{day}$ (arm 2); once-daily tacrolimus $0.3 \mathrm{mg} / \mathrm{kg} / \mathrm{day}$ (arm 3), all with mycophenolate mofetil and corticosteroids tapered to $\leq 5 \mathrm{mg}$ /day over 24 weeks; or once-daily tacrolimus $0.2 \mathrm{mg} / \mathrm{kg} /$ day with basiliximab, mycophenolate mofetil, and corticosteroids given only perioperatively (arm 4). About $10 \%$ of the patients had kidney transplants from living donors, while about $50 \%$ had extended-criteria donor kidneys. The primary endpoint was efficacy failure, a composite of graft loss, acute rejection detected on for-cause biopsy, or graft dysfunction (glomerular filtration rate $<40 \mathrm{~mL} / \mathrm{min}$ ) at 24 weeks. The majority of BPARs were mild to moderate and steroid-sensitive. Results showed that once-daily tacrolimus $0.2 \mathrm{mg} / \mathrm{kg}$ /day without induction therapy ( $\operatorname{arm} 2$ ) had similar efficacy to twice-daily tacrolimus $0.2 \mathrm{mg} / \mathrm{kg}$ /day (arm 1). Noninferiority of arm 3 and arm 4 against arm 1 was not confirmed. The incidence of diabetes and hypertension was comparable among the four groups. ${ }^{21}$

In a multicenter, open-label, controlled, noninferiority trial (Multicenter Evaluation of LCP Tacro tablets [MELT]) involving stable kidney transplant recipients, 324 patients were randomized to either remain on twice-daily tacrolimus or be converted to once-daily tacrolimus. The primary efficacy endpoint was efficacy failure that included death, graft failure, BPAR, or loss to follow-up within 12 months of follow-up. There were four cases of efficacy failure in both groups: two deaths due to cardiac arrest and two episodes of BPAR in the once-daily tacrolimus group, and one death due to lung cancer, two episodes of BPAR, and one loss to follow-up in the twice-daily tacrolimus group. All deaths were deemed unrelated to the study drug. Death-censored graft survival was $100 \%$ for both groups. Safety outcomes were similar between the two groups. ${ }^{22}$

In an open-label prospective observational study on Japanese kidney transplant recipients, 256 patients received once-daily tacrolimus at the onset of kidney transplant, while 106 were converted to once-daily tacrolimus post-transplant. The subjects were observed over 24 weeks. Among the subjects started on once-daily tacrolimus, the cumulative incidence of patient and graft survival was 98.2\%. Acute rejection rate was $16.1 \%$, of which $6.3 \%$ was steroid-resistant. Among the patients converted to once-daily tacrolimus posttransplant, there was one reported case of graft loss due to patient death from accident and one episode of steroidsensitive acute rejection. ${ }^{23}$

In an open-label, prospective, randomized controlled trial in Japanese living kidney transplant recipients, 102 patients were randomized to receive either once- $(n=50)$ or twicedaily $(n=52)$ tacrolimus at the onset of transplantation. 
Protocol kidney biopsies were performed at day 14 . Additional protocol kidney biopsies were performed within 6-12 months after transplantation. One-year patient and graft survival were $100 \%$ for both groups. Acute cellular rejection (at least Banff 1A) was reported in one patient in the oncedaily tacrolimus group and in five patients in the twice-daily tacrolimus group. Antibody-mediated rejection was noted in four patients in each group. Differences in BPAR incidences did not reach statistical significance. ${ }^{8}$

Another Japanese study looked into the safety and efficacy of once-daily tacrolimus by converting 33 kidney transplant patients, who were at least 1 year post-transplant, from twice- to once-daily tacrolimus. Serum creatinine, total cholesterol, uric acid, and fasting glucose were measured prior to and after conversion, all of which did not have any significant change with conversion. There were also no reported cases of rejection or infection after conversion to once-daily tacrolimus. ${ }^{24}$

In a multicenter study of 128 stable adult kidney transplant recipients converted from twice- to once-daily tacrolimus followed over 12 weeks, the once-daily formulation was found to be noninferior to twice-daily tacrolimus. Patient and graft survival were both $100 \%$. There were no reported cases of BPAR. Renal function remained stable after conversion to once-daily tacrolimus. The patients' blood pressure and hemoglobin $\mathrm{A}_{1 \mathrm{c}}$ also remained fairly unchanged. ${ }^{25}$

Slatinska et al performed a retrospective review of 589 stable adult kidney transplant recipients who were converted from twice- to once-daily tacrolimus. Concomitant immunosuppressive medications remained unchanged. Results showed that a milligram-to-milligram conversion from twice- to once-daily tacrolimus did not have a significant change in the total tacrolimus dose and trough levels. There was less interpatient variability of drug exposure observed after conversion to the once-daily formulation. At 12 months after conversion, patient survival was 99\%, while graft survival was $96.3 \%$. Renal function remained fairly stable after conversion, with glomerular filtration rate of about $46 \mathrm{~mL} / \mathrm{min}$ throughout the observation period. There were $14(2.4 \%)$ reported cases of BPAR: six were borderline rejection; two were Banff IA; three were Banff IB; and three were Banff IIA. Three developed BPAR within 3 months of conversion to once-daily tacrolimus. The cases of Banff IIA rejection had subtherapeutic tacrolimus trough levels $(<3 \mathrm{ng} / \mathrm{mL})$. All cases of BPAR were successfully treated with steroids. ${ }^{26}$

Wu et al conducted a retrospective cohort study on 199 stable Chinese kidney transplant recipients converted from twice- to once-daily tacrolimus. Two patients $(1 \%)$ developed BPAR after conversion, both of whom were noted to have subtherapeutic tacrolimus trough levels $(<3 \mathrm{ng} / \mathrm{mL}){ }^{27}$

In a retrospective study of 119 living donor kidney transplant recipients (90 patients on once-daily tacrolimus and 29 patients on twice-daily tacrolimus), protocol biopsies at 3 and 12 months were reviewed. Results showed that the frequency of subclinical rejection were similar between once- and twice-daily tacrolimus formulations. Interstitial fibrosis and tubular atrophy was less frequent and less severe in patients on once-daily tacrolimus at 12 months. ${ }^{28}$

Another retrospective review of preemptive kidney transplant recipients compared 27 patients who received oncedaily tacrolimus with 23 patients who received twice-daily tacrolimus at the onset of transplantation. Rejection was noted in one patient in the once-daily tacrolimus group and in three patients in the twice-daily tacrolimus group. All episodes of rejection responded to corticosteroid pulse treatment. No severe infections were reported, although BK viremia was noted in one patient in each group and cytomegalovirus (CMV) infection was noted in four patients in the once-daily group and seven patients in the twice-daily tacrolimus group. ${ }^{29}$

\section{Liver transplantation}

A double-blind, multicenter noninferiority trial ${ }^{30}$ involving 471 de novo liver transplant recipients showed that once-daily tacrolimus was noninferior to twice-daily tacrolimus as measured by BPAR rates at 6 and 12 months. Despite having numerically higher BPAR rates in the once-daily tacrolimus group, the values did not reach statistical significance. Treatment differences between once- and twice-daily tacrolimus also fell within the prespecified noninferiority margin of $15 \% .{ }^{30}$ Analysis of tacrolimus trough levels revealed no clear correlation of tacrolimus levels within the first 14 days post-transplant to acute rejection rates at 24 weeks on either formulation. ${ }^{5,30}$

A retrospective, observational, single-center study looked at 394 stable liver transplant patients converted to once-daily tacrolimus. Tacrolimus doses and trough levels, liver function test, and renal function were noted on the day of conversion, then at 1,6 , and 12 months post-conversion and every 6 months thereafter. During the observation period, liver and renal function remained stable. Blood pressure and lipid panel also remained unchanged post-conversion. The prevalence of diabetes increased slightly, but this did not reach statistical significance. BPAR was noted in seven patients post-conversion; BPAR in five of these patients was deemed mild, and two cases were moderate rejection. ${ }^{31}$ 
In a prospective, observational crossover study involving 61 liver transplant recipients, baseline clinical (body mass index, blood pressure, pulse rate, medication regimen) and laboratory (liver function test, serum creatinine, cholesterol, hemoglobin $A_{1 c}$ ) data were collected after 6 months of being on twice-daily tacrolimus. Subjects were then switched to once-daily tacrolimus, and the same data were collected at $3,6,9,12$, and 18 months. There was only one episode of acute rejection reported in a subject while on twice-daily tacrolimus. Thus, once- and twice-daily tacrolimus were deemed to have similar efficacy. ${ }^{32}$

Another prospective study on 65 liver transplant patients assessed the long-term efficacy and safety of conversion from twice- to once-daily tacrolimus. Laboratory parameters, metabolic disorders, and adverse events were monitored at 1 , $3,6,12$, and 24 months after conversion. Results showed that liver function, blood pressure, blood glucose, and cholesterol levels remained stable throughout 24 months of follow-up. Renal function was noted to improve at 24 months after conversion to once-daily formulation. There were no reported cases of acute rejection and adverse events. ${ }^{33}$

Valente et al converted 34 stable liver transplant recipients from twice- to once-daily tacrolimus and followed them over 21 months. Liver function and metabolic parameters remained stable post-conversion. Renal function was noted to increase at 6 and 12 months post-conversion. There were no reported cases of acute rejection and severe adverse events. ${ }^{34}$

\section{Summary}

Both head-to-head comparison and conversion trials conducted in de novo and stable transplant patients all showed that outcomes with once-daily tacrolimus are comparable to twice-daily tacrolimus. BPAR rates, although often higher with once-daily tacrolimus, were not statistically higher than with twice-daily tacrolimus. Patient survival and graft survival were similar between the two tacrolimus formulations. Once- and twice-daily tacrolimus also had similar effects on blood pressure, renal function, glucose metabolism, and lipid profile (Table 2).

\section{Safety and tolerability in long-term use}

Tacrolimus alters glucose and lipid metabolism. Thus, it has been suggested that avoidance of high tacrolimus $\mathrm{C}_{\max }$, often associated with the once-daily formulation, may decrease the incidence of hyperglycemia and hyperlipidemia among transplant patients. ${ }^{5}$ A study involving 20 stable kidney transplant patients showed a significant decrease in the incidence of hyperglycemia and hypertriglyceridemia following conversion from twice- to once-daily tacrolimus. ${ }^{35}$

In the randomized trial comparing both tacrolimus formulations, ${ }^{18}$ it was noted that constipation, gastroenteritis, paresthesia, fatigue, lower abdominal pain, ${ }^{5,18}$ pharyngitis, cytomegalovirus, and $\mathrm{BK}$ virus infections $(2.1 \%$ versus $0.3 \%, P=0.037)^{5,19}$ were more frequently reported in the once-daily tacrolimus group. On the other hand, incisionsite complications, graft dysfunction, urinary tract infection, cough, ${ }^{5,18}$ and bacterial infections were less frequently observed in the once-daily tacrolimus group., ${ }^{5,19}$

The MELT trial reported serious adverse events in $36(22 \%)$ patients in the once-daily tacrolimus group and in $26(16 \%)$ in the twice-daily tacrolimus group ( $P=$ nonsignificant). The most frequently reported adverse events were urinary tract infection, diarrhea, nasopharyngitis, upper respiratory tract infection, headache, edema, hypertension, and increased serum creatinine. ${ }^{22}$

In a Japanese prospective, observational study, common adverse drug reactions reported among the subjects started on once-daily tacrolimus included infection (23.6\%), most commonly with cytomegalovirus; renal disorder (6.8\%); and glucose intolerance (5.6\%). On the other hand, among the patients converted to once-daily tacrolimus post-transplant, common adverse reactions noted were infections, most often herpes zoster and nasopharyngitis. ${ }^{23}$

In the retrospective review of 589 adult kidney transplant patients converted from twice- to once-daily tacrolimus by Slatinska et al, there were 19 (3\%) reported cases of malignancy, six (1\%) cases of thrombotic microangiopathy, and three $(0.5 \%)$ cases of severe vascular changes, all of which were addressed by conversion of once-daily tacrolimus to sirolimus. The 19 cases of malignancy consisted of three cases of squamous cell skin cancer, two cases of basal cell skin cancer, one post-transplant lymphoproliferative disorder, seven genitourinary cancers, three respiratory cancers, two gastrointestinal cancers, and one thyroid cancer. Twenty-five $(4.2 \%)$ patients had worsening hypertension necessitating increase in antihypertensive regimen, while nine $(1.5 \%)$ patients with pre-transplant diabetes had persistent hyperglycemia and required conversion from oral hypoglycemic to insulin. Other frequently reported adverse events were urinary tract infection (3.7\%), liver enzyme elevation (3.4\%), anemia (2.7\%), leukopenia (2\%), and common warts $(2.6 \%){ }^{26}$

In the Chinese study of 199 kidney transplant recipients, reported adverse events were malaise $(3.5 \%)$, headache $(3 \%)$, oral ulcer $(1.5 \%)$, and skin rash $(1 \%)$. Only one patient 
developed an unspecified neoplasia. Seven patients noted deterioration in renal function and were converted back to twice-daily tacrolimus. ${ }^{27}$

In a prospective study, 40 kidney transplant recipients initially received twice-daily tacrolimus and then switched to once-daily tacrolimus. Laboratory parameters including creatinine clearance, homeostasis model assessment of insulin resistance, total cholesterol, low-density lipoprotein cholesterol, high-density lipoprotein cholesterol, triglycerides, homocysteine, and uric acid levels showed improvement after conversion to once-daily tacrolimus. The investigators concluded that conversion to once-daily tacrolimus could help improve cardiovascular risk factors among kidney transplant patients. ${ }^{36}$

In a prospective study of 26 kidney transplant recipients who were converted from twice to once daily tacrolimus. The homeostasis model assessment of $\beta$-cell function (HOMA- $\beta$ ) was calculated before and after conversion in order to evaluate the effect of once-daily both tacrolimus formulations on glucose metabolism. Results showed that HOMA- $\beta$ increased while hemoglobin $A_{1 c}$ decreased significantly after 4 weeks of conversion to once-daily tacrolimus. The same effects were noted at 24 weeks after conversion, supporting the premise that conversion to once-daily tacrolimus may improve pancreas $\beta$-cell function and glucose tolerance. The investigators inferred that this effect may be related to the lower $\mathrm{C}_{\max }$ achieved with once-daily tacrolimus. ${ }^{37}$

In the single-center study by Dumortier et al, adverse events reported on liver transplant patients on once-daily tacrolimus were tremor, diarrhea, cutaneous eruption, oral drought, headache, insomnia, hypertension, diabetes, asthenia, and erectile dysfunction. The above events triggered conversion of the patients back to the twice-daily formulation. ${ }^{31}$

Weiler et al noted no significant differences in the rate of hypertension, diabetes, hypercholesterolemia, hyperuricemia, and renal insufficiency among liver transplant recipients who were converted from twice- to once-daily tacrolimus. Hemoglobin $\mathrm{A}_{1 \mathrm{c}}$ was noted to be significantly higher while on once-daily tacrolimus. ${ }^{32}$

In a study on lung transplant patients converted to oncedaily tacrolimus, frequently reported adverse events were cough, pharyngitis, bronchitis, diarrhea, edema, and tremors. There were no reports of renal dysfunction or new cases of diabetes post-conversion. ${ }^{12}$

\section{Summary}

Safety studies on once-daily tacrolimus showed that most reported adverse events included metabolic (hyperglycemia, dyslipidemia), gastrointestinal (diarrhea, nausea), neurologic (tremors, headaches), and infectious (bacterial urinary tract infection, CMV) complications. Most adverse events were mild to moderate in severity. There was discordance in reports on the effect of once-daily tacrolimus on glucose metabolism, in that some studies showed improvement in pancreas function while others showed increase in hemoglobin $\mathrm{A}_{1 \mathrm{c}}$ and insulin requirement. For the most part, studies that showed improvement in glucose metabolism with once-daily tacrolimus attribute this benefit from the lower $\mathrm{C}_{\max }$ achieved with the once-daily formulation. Whether benefits accrued with once-daily tacrolimus are free of the double-edged risk of rejection with underexposure, and complications such as $\mathrm{BK}$ virus infections with overexposure, demand further study.

\section{Patient-reported outcomes and treatment adherence}

In this section, we review the impact of once-daily tacrolimus on several domains measuring patient reported outcomes and measured treatment adherence. In a prospective cohort study conducted to assess patient satisfaction on once-daily tacrolimus, 75 kidney transplant recipients were converted milligram to milligram from twice- to once-daily tacrolimus. Other medications were also switched to a once-daily formulation. The subjects' treatment satisfaction was then evaluated using the Treatment Satisfaction Questionnaire for Medication (TSQM). Results showed that, 3 weeks after conversion, convenience scores significantly increased. However, TSQM scores for effectiveness, tolerability, and global satisfaction did not change significantly. Self-reported adherence did increase while missed drug doses decreased significantly. ${ }^{2}$

In a randomized, multicenter, controlled trial involving 219 kidney transplant recipients, 145 received once-daily tacrolimus and 74 received twice-daily tacrolimus. Electronic monitoring was used to document drug intake for 6 months. Results showed that the once-daily tacrolimus group had better persistence and implementation with medication and less missed drug doses compared to the twice-daily tacrolimus group. The once-daily tacrolimus group also had more accurate and timely drug dosing. ${ }^{38}$

In a Japanese observational study reported by Takahashi et al, there was no noted difference in compliance among kidney transplant patients converted from twice- to oncedaily tacrolimus, with $98 \%$ of the subjects having at least a $90 \%$ compliance rate. ${ }^{23}$

Medication compliance among 63 liver transplant patients was assessed while on twice-daily tacrolimus for 
6 months and then after switching to once-daily tacrolimus for 6 months. Patients were grouped based on time from liver transplantation: 6 months to 2 years, 2-5 years, and greater than 5 years after transplant. The electronic monitoring system revealed that dosing, taking, and timing compliance rates were higher in the once-daily tacrolimus group. There were no significant differences in compliance rates in the groups based on time from liver transplantation. Finally, quality of life as measured by the Short Form-36 (SF-36) questionnaire was better with once-daily dosing. ${ }^{39}$

Valente et al looked into medication adherence in liver transplant recipients before and after conversion from twice- to once-daily tacrolimus. Visual analog scale showed a baseline adherence of $86 \%$, which increased to $90 \%$ postconversion. Based on the Basel Assessment of Adherence with Immunosuppressive Medication Scale (BAASIS), abnormal drug intake behavior was noted by $45 \%$ of subjects prior to conversion. This improved to $35 \%$ post-conversion to oncedaily formulation. All patients converted to once-daily tacrolimus preferred to remain on the once-daily formulation. ${ }^{34}$

A study of 72 stable heart transplant patients converted from twice-daily cyclosporine or tacrolimus to once-daily tacrolimus showed a significant decrease in overall nonadherence, as measured by BAASIS 8 months after conversion. Medication adherence also increased in about $60 \%$ of the subjects, attributed mostly to timely drug intake and fewer missed doses. In this study, conversion back to twice-daily calcineurin inhibitor was driven mostly by mild gastrointestinal symptoms while taking once-daily tacrolimus. ${ }^{40}$

\section{Summary}

Studies on medication compliance showed that patients converted to once-daily tacrolimus had improved adherence in terms of drug taking, timing, and dosing. There was also a trend toward decrease in missed doses with once-daily tacrolimus.

\section{Discussion}

Pharmacokinetic studies of bioequivalence suggest that oncedaily tacrolimus is comparable to twice-daily tacrolimus. However, some efficacy studies show that BPAR may be higher with once-daily tacrolimus. This relationship between once-daily tacrolimus and acute rejection may be modifiable based on knowledge that tacrolimus trough levels achieved with once-daily tacrolimus tend to be lower compared with twice-daily tacrolimus. Moreover, trough levels extrapolated from twice-daily dosing schedules may not be the optimal way to monitor the once-daily formulation. Despite the higher rates of acute rejection, a major clinically relevant advantage with once-daily tacrolimus is decreased intrapatient inter-occasion PK variability. Since variability in levels of tacrolimus contributes to increased risk of acute rejection, late allograft rejection, transplant glomerulopathy, and graft loss, ${ }^{41}$ oncedaily tacrolimus could be potentially advantageous, especially among patients who could have high CYP3A5 expression. Compliance with once-daily tacrolimus is also apparently higher in studies of limited duration, but long-term experience in the context of routine clinical practice is yet to accrue. At the time of this writing, it appears that a regimen that includes once-daily tacrolimus could represent a viable option for immunosuppression, either at the onset of transplantation or for conversion from the twice-daily formulation. The benefit of using once-daily tacrolimus lies in the potential for improving adherence to medications of transplant patients, especially in those who have issues with dosing schedule and complex medication regimen. Improvement in immunosuppression medication adherence could be intuitively inferred to decrease risk of allograft rejection and consequently improve allograft outcomes. The potential benefit of once-daily tacrolimus in improving cardiovascular risk factors, including glucose metabolism, lipid profile, uric acid, and homocysteine, is suggested in small studies. However, this finding needs further study in the context of sustained freedom from acute rejection.

This review pertains to cumulative studies conducted on different formulations of once-daily tacrolimus and does not endorse a particular brand. Our review of existing studies on once-daily tacrolimus suggests that an initial dose of $0.2-0.3$ $\mathrm{mg} / \mathrm{kg}$ /day within 24 hours of kidney transplantation and $0.1-$ $0.2 \mathrm{mg} / \mathrm{kg} /$ day within $12-18$ hours of liver transplantation for de novo patients, ${ }^{5}$ or a milligram-to-milligram conversion from twice-daily tacrolimus among stable transplant patients, may provide equivalent tacrolimus exposure to that achieved with the twice-daily formulation. Currently recommended drug monitoring is based on trough level measured from a sample of whole blood obtained 24 hours after the last dose of once-daily tacrolimus. Therapeutic drug monitoring is very important, especially given reports of lower achieved $\mathrm{C}_{\max }$ and trough levels with once-daily tacrolimus. It is prudent to increase the dose of once-daily tacrolimus if the desired target level is not achieved in order to prevent increased risk of acute rejection. Finally, any acute changes in allograft function should prompt immediate investigation.

\section{Conclusion}

Once-daily tacrolimus clearly has potential benefits in clinical transplantation in terms of simplifying immunosuppressive 
regimens, improving medication compliance, decreasing PK variability, and consequently, reducing the risk of acute rejection. As once-daily tacrolimus becomes more frequently utilized in clinical practice, more studies on efficacy, compliance, and safety with long-term use need to be conducted in order to better understand drug effects and refine drug administration among transplant patients.

\section{Disclosure}

TRS has received research grant support from Astellas. The authors report no other conflicts of interest in this work.

\section{References}

1. Kaplan B, Srinivas TR, Meier-Kriesche HU. Factors associated with long-term renal allograft survival. Ther Drug Monit. 2002;24(1): 36-39.

2. van Boekel GA, Kerkhofs CH, Hilbrands LB. Treatment satisfaction in renal transplant patients taking tacrolimus once daily. Clin Ther. 2013;35(11):1821-1829. e1.

3. United States Organ Transplantation. OPTN/SRTR 2012 Annual Data Report [webpage on the Internet]. US Department of Health and Human Services; 2014. Available from: http://srtr.transplant.hrsa.gov/ annual_reports/2012/flash/2012_SRTR_ADR/index.html. Accessed June 6, 2014.

4. Ho ET, Wong G, Craig JC, Chapman JR. Once-daily extended-release versus twice-daily standard-release tacrolimus in kidney transplant recipients: a systematic review. Transplantation. 2013;95(9): 1120-1128.

5. Barraclough KA, Isbel NM, Johnson DW, Campbell SB, Staatz CE. Once- versus twice-daily tacrolimus: are the formulations truly equivalent? Drugs. 2011;71(12):1561-1577.

6. Tsunashima D, Kawamura A, Murakami M, et al. Assessment of tacrolimus absorption from the human intestinal tract: open-label, randomized, 4-way crossover study. Clin Ther. 2014;36(5):748-759.

7. First MR, Fitzsimmons WE. Modified release tacrolimus. Yonsei Med J. 2004;45(6):1127-1131.

8. Tsuchiya $\mathrm{T}$, Ishida $\mathrm{H}$, Tanabe $\mathrm{T}$, et al. Comparison of pharmacokinetics and pathology for low-dose tacrolimus once-daily and twice-daily in living kidney transplantation: prospective trial in once-daily versus twice-daily tacrolimus. Transplantation. 2013;96(2):198-204.

9. Gaber AO, Alloway RR, Bodziak K, Kaplan B, Bunnapradist S. Conversion from twice-daily tacrolimus capsules to once-daily extended-release tacrolimus (LCPT): a phase 2 trial of stable renal transplant recipients. Transplantation. 2013;96(2):191-197.

10. Alloway RR, Eckhoff DE, Washburn WK, Teperman LW. Conversion from twice daily tacrolimus capsules to once daily extended-release tacrolimus (LCP-Tacro): phase 2 trial of stable liver transplant recipients. Liver Transpl. 2014;20(5):564-575.

11. Min SI, Ha J, Kang HG, et al. Conversion of twice-daily tacrolimus to once-daily tacrolimus formulation in stable pediatric kidney transplant recipients: pharmacokinetics and efficacy. Am J Transplant. 2013;13(8): 2191-2197.

12. Lapeyraque AL, Kassir N, Théorêt Y, et al. Conversion from twice- to once-daily tacrolimus in pediatric kidney recipients: a pharmacokinetic and bioequivalence study. Pediatr Nephrol. 2014;29(6):1081-1088.

13. Méndez A, Berastegui C, López-Meseguer M, et al. Pharmacokinetic study of conversion from tacrolimus twice-daily to tacrolimus once-daily in stable lung transplantation. Transplantation. 2014;97(3):358-362.

14. Stifft F, Stolk LM, Undre N, van Hooff JP, Christiaans MH. Lower variability in 24-hour exposure during once-daily compared to twice-daily tacrolimus formulation in kidney transplantation. Transplantation. Epub December 13, 2013.
15. Macphee IA, Fredericks S, Mohamed M, et al. Tacrolimus pharmacogenetics: the CYP3A $5 * 1$ allele predicts low dose-normalized tacrolimus blood concentrations in whites and South Asians. Transplantation. 2005;79(4):499-502.

16. Niioka T, Kagaya H, Miura M, et al. Pharmaceutical and genetic determinants for interindividual differences of tacrolimus bioavailability in renal transplant recipients. Eur J Clin Pharmacol. 2013;69(9): 1659-1665.

17. Wu MJ, Chang $\mathrm{CH}$, Cheng $\mathrm{CY}$, et al. Reduced variability of tacrolimus trough level in once-daily tacrolimus-based Taiwanese kidney transplant recipients with high-expressive genotype of cytochrome P450 3 A5. Transplant Proc. 2014;46(2):403-405.

18. Silva HT Jr, Yang HC, Abouljoud M, et al. One-year results with extended-release tacrolimus/MMF, tacrolimus/MMF and cyclosporine/ MMF in de novo kidney transplant recipients. Am J Transplant. 2007;7(3):595-608.

19. Krämer BK, Charpentier B, Bäckman L, et al; Tacrolimus Prolonged Release Renal Study Group. Tacrolimus once daily (ADVAGRAF) versus twice daily (PROGRAF) in de novo renal transplantation: a randomized phase III study. Am J Transplant. 2010;10(12): 2632-2643.

20. Srinivas TR, Kaplan B, Meier-Kriesche HU. The noninferiority trial: don't don't do it. Am J Transplant. 2010;10(12):2571-2573.

21. Albano L, Banas B, Klempnauer JL, Glyda M, Viklicky O, Kamar N; Optimising immunoSuppression After Kidney transplantation with ADVAGRAF Study Group. OSAKA trial: a randomized, controlled trial comparing tacrolimus $\mathrm{QD}$ and $\mathrm{BD}$ in kidney transplantation. Transplantation. 2013;96(10):897-903.

22. Bunnapradist S, Ciechanowski K, West-Thielke P, et al. Conversion from twice-daily tacrolimus to once-daily extended release tacrolimus (LCPT): the phase III randomized MELT trial. Am J Transplant. 2013;13(3):760-769.

23. Takahashi K, Abe R, Usuki S, So M. Safety and efficacy of once-daily modified-release tacrolimus in kidney transplant recipients: interim analysis of multicenter postmarketing surveillance in Japan. Transplant Proc. 2014;46(2):406-410.

24. Nakamura Y, Hama K, Katayama H, et al. Safety and efficacy of conversion from twice-daily tacrolimus (prograf) to once-daily prolonged-release tacrolimus (graceptor) in stable kidney transplant recipients. Transplant Proc. 2012;44(1):124-127.

25. Lauzurica R, Morales JM, van Hooff J, Study Investigators. Renal function and safety in stable kidney transplant recipients converted from immediate-release to prolonged-release tacrolimus. Transpl Int. 2012;25(1):48-55.

26. Slatinska J, Rohal T, Wohlfahrtova M, Viklicky O. Long-term follow-up of stable kidney transplant recipients after conversion from tacrolimus twice daily immediate release to tacrolimus once-daily prolonged release: a large single-center experience. Transplant Proc. 2013;45(4): 1491-1496.

27. Wu SW, Tsai HC, Tsai PY, Hung TW, Chang HR, Lian JD. Conversion to prolonged release tacrolimus formulation in stable kidney transplant recipients. Swiss Med Wkly. 2013;143:w13850.

28. Masutani K, Tsuchimoto A, Haruyama N, et al. Protocol biopsy findings in living donor kidney transplant patients treated with once-daily or twice-daily tacrolimus formulation. Transplant Proc. 2014;46(2):395-399.

29. Kitada H, Okabe Y, Nishiki T, et al. One-year follow-up of treatment with once-daily tacrolimus in de novo renal transplant. Exp Clin Transplant. 2012;10(6):561-567.

30. Trunečka P, Boillot O, Seehofer D, et al; Tacrolimus Prolonged Release Liver Study Group. Once-daily prolonged-release tacrolimus (ADVAGRAF) versus twice-daily tacrolimus (PROGRAF) in liver transplantation. Am J Transplant. 2010;10(10):2313-2323.

31. Dumortier J, Guillaud O, Boillot O. Conversion from twice daily tacrolimus to once daily tacrolimus in long-term stable liver transplant recipients: a single-center experience with 394 patients. Liver Transpl. 2013;19(5):529-533.

32. Weiler N, Thrun I, Eberlin M, et al. Tacrolimus effects and side effects after liver transplantation: is there a difference between immediate and extended release? Transplant Proc. 2013;45(6):2321-2325. 
33. Giannelli V, Rossi M, Giusto M, et al. Conversion from twice-daily to once-daily Tacrolimus administration in liver transplant patient: results of long term follow-up. Eur Rev Med Pharmacol Sci. 2013;17(20): 2718-2720.

34. Valente G, Rinaldi L, Sgambato M, Piai G. Conversion from twice-daily to once-daily tacrolimus in stable liver transplant patients: effectiveness in a real-world setting. Transplant Proc. 2013;45(3):1273-1275.

35. Meçule A, Poli L, Nofroni I, et al. Once daily tacrolimus formulation: monitoring of plasma levels, graft function, and cardiovascular risk factors. Transplant Proc. 2010;42(4):1317-1319.

36. Sessa A, Esposito A, Iavicoli G, et al. Cardiovascular risk factors in renal transplant patients after switch from standard tacrolimus to prolongedrelease tacrolimus. Transplant Proc. 2012;44(7):1901-1906.

37. Uchida J, Iwai T, Kabei K, et al. Effects of conversion from a twice-daily tacrolimus to a once-daily tacrolimus on glucose metabolism in stable kidney transplant recipients. Transplant Proc. 2014;46(2):532-536.
38. Kuypers DR, Peeters PC, Sennesael JJ, et al. Improved adherence to tacrolimus once-daily formulation in renal recipients: a randomized controlled trial using electronic monitoring. Transplantation. 2013;95(2): 333-340.

39. Eberlin M, Otto G, Krämer I. Increased medication compliance of liver transplant patients switched from a twice-daily to a once-daily tacrolimus-based immunosuppressive regimen. Transplant Proc. 2013;45(6):2314-2320.

40. Doesch AO, Mueller S, Akyol C, et al. Increased adherence eight months after switch from twice daily calcineurin inhibitor based treatment to once daily modified released tacrolimus in heart transplantation. Drug Des Devel Ther. 2013;7:1253-1258.

41. Sapir-Pichhadze R, Wang Y, Famure O, Li Y, Kim SJ. Timedependent variability in tacrolimus trough blood levels is a risk factor for late kidney transplant failure. Kidney Int. 2014;85(6): $1404-1411$.

\section{Publish your work in this journal}

Drug Design, Development and Therapy is an international, peerreviewed open-access journal that spans the spectrum of drug design and development through to clinical applications. Clinical outcomes, patient safety, and programs for the development and effective, safe, and sustained use of medicines are a feature of the journal, which has also been accepted for indexing on PubMed Central. The manuscript management system is completely online and includes a very quick and fair peer-review system, which is all easy to use. Visit http://www.dovepress.com/testimonials.php to read real quotes from published authors.

Submit your manuscript here: http://www.dovepress.com/drug-design-development-and-therapy-journal 